\title{
A CONDITION FOR SIMPLE RING IMPLYING FIELD II
}

\author{
CHEN-TE YEN
}

\begin{abstract}
It is shown that if $R$ is a simple ring with identity 1 and with a nonzero idempotent $e$ and satisfies the condition $\left(P_{2}\right)_{e}$ :

$\left(P_{2}\right)_{e} \quad$ If $e-\left(a_{1} b_{1}+a_{2} b_{2}\right)$ is a right (left) zero

divisor in $R$, then so is $e-\left(b_{1} a_{1}+b_{2} a_{2}\right)$.

then $R$ is a field.Thus if $R$ is a simple ring then $e R e$ is a field for every nonzero idempotent $e$ in $R$ if it exists and $e R e$ satisfies $\left(P_{2}\right)_{e}$. We also discuss the above property for the simple ring case by eliminating the identity 1 .
\end{abstract}

\section{Introduction}

Throughout the note $R$ will denote an associative ring. An element a in $R$ is called a right (left) zero divisor ([2], p. 88) if there exists a nonzero element $b$ of $R$ such that $b a=0(a b=0) ; a$ is a zero divisor if it is a right and a left zero divisor. Clearly 0 is a zero divisor if $R$ has more than one element.

Let $R$ be a ring with a nonzero idempotent $e$, i.e., $e^{2}=e$, and let $n$ be a positive integer. We define the property $\left(P_{n}\right)_{e}$ as follows:

$\left(P_{n}\right)_{e} \quad$ If $e-\sum_{i=1}^{n} a_{i} b_{i}$ is a right (left) zero divisor

in $R$, then so is $e-\sum_{i=1}^{n} b_{i} a_{i}$.

Let $R$ satisfy $\left(P_{n}\right)_{e}$. Then $R$ satisfies $\left(P_{m}\right)_{e}$ for every $m=1,2, \cdots, n-1$. It is easy to show that if $f$ is an isomorphism from $R$ onto a ring $S$ then $f(e)^{2}=f\left(e^{2}\right)=f(e)$ and $S$ satisfies the property $\left(P_{n}\right)_{f(e)} . R$ is called simple ([2], p. 107) if $R^{2}=R$ and the only ideals in $R$ are 0 and $R$. Note that if $R$ is a simple ring then eRe is also a simple ring with identity $e$.

Received March 10, 1993; revised April 29, 1993.

1991 Mathematics Subject Classification. Primary 16A99.

Key words and phrases. Jacobson radical, local ring, semiprime ring, simple ring, field. 
$R$ is called semiprime if the only ideal of $R$ which squares to zero is the zero ideal. Note that if $R$ is a semiprime ring then $R x \neq 0$ and $x R \neq 0$ for every nonzero element $x$ in $R$. We show that if $R$ is a semiprime ring then every right (left) identity of $R$ is an identity.

Let $R$ have identity 1 . We shall denote the property $\left(P_{n}\right)_{1}$ by $\left(P_{n}\right)$. It is easy to prove that $R$ always satisfies $\left(P_{1}\right)$. As the proof of lemma 1 of [4], we have that $R$ satisfies $\left(P_{n}\right)$ for all positive integers $n$ if and only if $R$ satisfies $\left(P_{2}\right)$. Noncommutative rings satisfying $\left(P_{2}\right)$ actually exist, e.g., the Examples 1 and 2 of [3].

The purpose of this note is to prove the following theorem: if $R$ is a simple ring with identity 1 and with a nonzero idempotent $e$ and satisfies $\left(P_{2}\right)_{e}$ then $R$ is a field. In particular, any simple ring with identity 1 satisfying $\left(P_{2}\right)$ must be a field. Thus we obtain the following result: if $R$ is a simple ring then $e R e$ is a field for every nonzero idempotent $e$ in $R$ if it exists and $e R e$ satisfies $\left(P_{2}\right)_{e}$. We ask whether the hypothesis " $R$ has identity 1 " can be eliminated in the above theorem.

Let $R$ be a ring. We shall denote the Jacobson radical by $J(R) . R$ is called local if $R$ has the unique maximal ideal. We also discuss the above property for the simple ring case by eliminating the identity 1 . For the related results, see [5] - [8].

\section{Results}

We begin with two easy known results.

Proposition 1. If $R$ is a semiprime ring, then every right (left) identity of $R$ is an identity.

Proof. Let $e$ be a right identity of $R$. Assume that $x$ is a nonzero element in $R$. Since $R$ is semiprime, $R x \neq 0$. Thus, we obtain $R(e x)=(R e) x=R x \neq 0$. Hence, $e x \neq 0$. Thus, $e(e x-x)=0$ implies $e x=x$. So, $e$ is an identity of $R$. The proof of the other (left) case is similar.

Proposition 2. If $R$ is a ring with identity 1, then $R$ satisfies $\left(P_{1}\right)$.

Proof. Assume that $1-a b$ is not a right zero divisor in $R$. Let $c$ be an element of $R$ such that $c(1-b a)=0$. Then $c=c b a$ and $c b(1-a b)=c(1-b a) b=0$. By assumption, $c b(1-a b)=0$ implies $c b=0$. Thus, $c=c b a=0$. Hence $1-b a$ is not a right zero divisor. The proof of the other (left) case is similar.

As the proof of Lemma 1 of [4], we have the

Lemma 1 . If $R$ is a ring with identity 1 , then $R$ satisfies $\left(P_{n}\right)$ for all positive integers $n$ if and only if $R$ satisfies $\left(P_{2}\right)$.

Lemma 2. If $R$ is a ring with identity 1 and satisfies $\left(P_{2}\right)$, then every commutator $x y-y x$ is a zero divisor for all $x, y$ in $R$.

Proof. Since $R$ satisfies $\left(P_{2}\right)$, by Lemma $1, R$ satisfies $\left(P_{n}\right)$ for all positive integers 
n. For all $x, y$ in $R$, we have $1-(1-(x y-x y))=0$. Thus by $\left(P_{3}\right), x y-y x=$ $1-(1-(x y-y x))$ is a zero divisor, as desired.

Lemma 3. If $R$ is a simple ring with identity 1 and satisfies $\left(\mathbb{P}_{2}\right)$, then $R$ is a field.

Proof. Let $c$ be a nonzero element of $R$. We show that $c$ is neither a right nor a left zero divisor. Since $R$ is simple, $R c R=R$. Thus, there exist $a_{i}, b_{i}$ in $R, i=1,2, \cdots, m$, such that $\sum_{i=1}^{m} a_{i} c b_{i}=1$. Let $d=\sum_{i=1}^{m} b_{i} a_{i}$. By Lemma $1, R$ satisfies $\left(P_{n}\right)$ for all positive integers $n$.

Hence using this twice, $1=1-\left(1-\sum_{i=1}^{m} a_{i} c b_{i}\right)$ implies $c d=1-(1-c d)$ and $d c=$ $1-(1-d c)$ are neither right nor left zero divisors. It follows from these that $c$ is neither a right nor a left zero divisor.

By Lemma 2, $x y-y x$ is a zero divisor for all $x, y$ in $R$. Thus by the result above, $x y=y x$. Hence $R$ is a commutative simple ring with identity. So, $R$ is a field, as desired.

Using Lemma 3, we obtain the

Corollary. If $R$ is a simple ring, then eRe is a field for every nonzero idempotent $e$ in $R$ if it exists and eRe satisfies $\left(P_{2}\right)_{e}$.

Theorem 1. If $R$ is a simple ring with identity 1 and with a nonzero idempotent $e$ and satisfies $\left(\mathbb{P}_{2}\right)_{e}$, then $R$ is a field.

Proof. Since $R$ is a simple ring, $e R e$ is a simple ring with identity $e$ and satisfies $\left(P_{2}\right)_{e}$. Thus by Lemma 3 , eRe is a field. Hence we obtain exeye $=($ exe $)($ eye $)=$ (eye) (exe) = eyexe for all $x, y$ in $R$. Using ([1], p. 24, Corollary), $R$ is isomorphic to the ring of all $m \times m$ matrices over a field $F$ for some positive integer $m$. Let $f: R \rightarrow F_{m}$ be this isomorphism. Suppose $m>1$. Because of $f(e)^{2}=f(e)$, by the well known result of linearr algebra there exists an invertible matrix $A$ in $F_{m}$ such that $A f(e) A^{-1}=\sum_{i=1}^{k} e_{i i}$ for some positive integer $k$. Let $B=\sum_{i=1}^{k} e_{i i}$. Therefore we have that $R \cong F_{m} \cong A F_{m} A^{-1}=F_{m}$. So, $F_{m}$ satisfies the property $\left(P_{2}\right)_{B}$. Thus by Lemma $1, F_{m}$ satisfies $\left(P_{n}\right)_{B}$ for all positive integers $n$. If $k=m$, then $f(e)=A^{-1} B A=1$ and so $e=1$. Hence by Lemma $3, R$ is a field, so we may assume $k<m$. Then $B-\sum_{i=k+1}^{m}\left(-e_{1 i}\right) e_{i 1}$ is a zero divisor in $F_{m}$. Since $F_{m}$ satisfies $\left(P_{m-k}\right)_{B^{\prime}}$ this implies $1=B-\sum_{i=k+1}^{m} e_{i 1}\left(-e_{1 i}\right)$ is a zero divisor, a contradiction. Thus $m=1$. So, $R \cong F$. Hence $R$ is a field.

In Theorem 1, if we eliminate the identity 1 then we obtain the

Theorem 2. If $R$ is a simple ring with a nonzero idempotent e and satisfies $\left(P_{2}\right)_{e}$, then either $R$ is a field; or $e R$ and $R$ e are local rings which consist of zero divisors and $e R / J(e R)$ and $R e / J(R e)$ are fields.

Proof. Using Theorem $1, e R e$ is a field. By symmetry, we only consider the right ideal $e R$ of $R$. Clearly, $e R$ has a left identity $e$. Assume that $A$ is a nonzero right ideal of $e R$ and $a e \neq 0$ for some a in $A$. Then there exists an element $c$ of $R$ such that aece $=(e a e)(e c e)=e$, since $e R e$ is a field. Thus, $e=$ aece $\in A$ and so $A=e R$. Hence, 
either $e R$ is a simple ring or $A e=0$ for every proper right ideal $A$ of $e R$.

Assume that $e R$ is a simple ring. By Proposition $1, e$ is the identity of $e R$. Thus, we have

$$
\text { exe }=(e x) e=e(e x)=e x \text { for all } x \text { in } R \text {. }
$$

Let $B=\{b \in R: e b=0\}$. Obviously, $B$ is a right ideal of $R$. Assume that $b \in B$ and $x \in R$. Then by $(*)$, we obtain $e x b=(e x e) b=e x(e b)=0$. Hence $B$ is an ideal of $R$. By the simplicity of $R$ and $e R \neq 0$, we get $B=0$. Thus, $e(x e-e x e)=0$ implies $x e=e x e$. By $(*), e x=e x e=x e$. Hence $e$ is central. So, $e R=R$. Therefore, $R=e R=e R e$ is a field.

Assume that $A e=0$ for every proper right ideal $A$ of $e R$. Let $I$ be the sum of all proper right ideals of $e R$. Then $I$ is the unique maximal right ideal in $R$. Thus $I=J(e R)$. Hence $e R$ is a local ring. For all $x$ in $R$, since $x e=e-(e-x e)$ is a right zero divisor, by $\left(P_{2}\right)_{e}, e x=e-(e-e x)$ is also a right zero divisor. Clearly, ex is a left zero divisor. Thus $e R$ consists of zero divisors. As above, we can easily show that $e R / J(e R)$ is a field.

\section{References}

[1] I. N. Herstein, Rings with involution, Univ. of Chicago press (Chicago, 1976).

[2] N. Jacobson, Basic Algebra. I, W. H. Freeman (San Francisco, 1974).

[3] C. T. Yen, "On the commutativity of primary rings," Math. Japon., 25(1980), 449-452.

[4] C. T. Yen, "A condition for simple ring implying field," unpublished manuscript.

[5] C. T. Yen, "On rings satisfying both of $1-a b c$ and $1-c b a$ being invertible or none," Tamkang $J$. Math., 24, No. 3(1993), 317-321.

[6] C. T. Yen, "A note on simple rings satisfying a condition," unpublished manuscript.

[7] C. T. Yen, "A condition for simple ring implying field III," submitted.

[8] C. T. Yen, "Associative rings with some conditions," unpublished manuscript. 